\section{Round and about the Universe}

\section{Michael Rowan-Robinson}

Light From the Depths of Time. By Rudolf Kippenhahn. Springer-Verlag: 1987. Pp. 262. Pbk DM54.

I FIRST heard Rudolf Kippenhahn lecture at a summer school 20 years ago, and I remember the humour, clarity and imagination that he brought to his course on stellar structure. It is no surprise to me, therefore, that he should be capable of writing a really excellent popular book on astronomy. More surprising, perhaps, is the subject, cosmology. In fact the scope of Light From the Depths of Time is very wide - the structure and evolution of galaxies, the distance scale, special and general relativity, active galaxies and quasars, as well as the history of the Big Bang and the large-scale structure of the Universe.

The original, German edition of the book appeared in 1984 and the cosmological story presented dates from the early 1980s. Since then several developments have profoundly altered the cosmological consensus - grand unified theories, inflation, cold dark matter, biased galaxy formation - so this is not an account for those who want to read about the new synthesis of particle physics and cosmology. But this detracts little from the book's value as an introduction to cosmology and as an enjoyable read.

The qualities required of a good popular science book are imagination, a human dimension and a philosophical perspective. The main imaginative device employed by Kippenhahn is a series of dreams of one Herr Meyer, the man on the Munich omnibus. This device, frankly borrowed from George Gamow's $\mathrm{Mr}$ Tompkins, is very effective and takes the book to the brink of the truly inspiring (I do not fully understand why books by contemporary scientists are not able to cross that bridge of inspiration in the way that, say, James Jeans did, and that a writer such as Calvino does). Herr Meyer's dreams also add a human touch to the book, as, more conventionally, do the biographical anecdotes about astronomers.

The main virtues of Light From the Depths of Time are its vivid style and imaginative presentation, and these take it beyond the coffee-table type of book crammed with pictures which clogs up the astronomy shelf in bookshops (you know, that shelf underneath the eight shelves of astrology titles). It does not have a strong philosophical perspective, in the sense of a reason why the book had to be written. In his final pages, Kippenhahn inclines to the view that the tenets of physics are projections of our own minds onto the Universe rather than inexorable laws that determine the course of events. I would like to have seen this argument developed further, perhaps at the expense of omitting the concluding device of compressing the history of the Universe into one year, with human history occupying the last fifteen seconds. This is simply Pascal's terror

\section{Star attraction}

\section{Stephen P. Maran}

The Quest for SS433: The Discovery of the Astronomical Phenomenon of the Century. By David H. Clark. Adam Hilger: 1986. Pp.206. £9.95.

"Look at what we've been monitoring for Bruce" was an observer's excited request when I visited the control room of the 4-metre telescope at Kitt Peak National Observatory in October, 1978. "Bruce" was Bruce Margon, a young astronomer then at the University of California, Los Angeles, who had convinced several investigators to take spectra of the unusual star SS433 with telescopes in northern and southern California, and in Arizona, at every opportunity.

The spectra I saw on that occasion showed astonishing changes in the positions of emission lines from night to night. News of them was to burst upon the scientific world shortly thereafter, when Margon's group announced their discovery. SS433 is a bizarre star, which appears to have oppositely directed jets that spew matter into space at a quarter of the speed of light while they precess, tracing conical patterns in the sky once every 164 days. The jets arise from the compact member, perhaps a black hole, of an interacting binary system which has an orbital period of 13 days.

More than eight years have now passed, during which SS433 must have been inspected with every suitable optical and radio telescope in the world, and several satellites as well (Exosat and TENMA among them). Fresh details about its complex emissions are announced regularly, but we still have no confirmed reports of any truly comparable star, in our Galaxy or elsewhere. To the extent that there are known analogues for SS433, they are not stars but the active galactic nuclei that produce the powerful jets found in Cygnus $\mathbf{A}$ and other radio galaxies, and in quasars. SS433, on a stellar scale, is the closest example of the much more energetic phenomena in the hearts of those distant objects.

It was the British astronomer David $\mathrm{H}$. Clark and his colleague Paul Murdin who, in June 1978, discovered the peculiar before the infinite spaces and times of the Universe. But in a single human lifetime there is already more significance, more consciousness, more meaning than in all those vast, cold, empty spaces and times that exist without us.

Michael Rowan-Robinson is Reader in Astronomy in the School of Mathematical Sciences, Queen Mary College, Mile End Road, London E1 $4 N S$, UK.

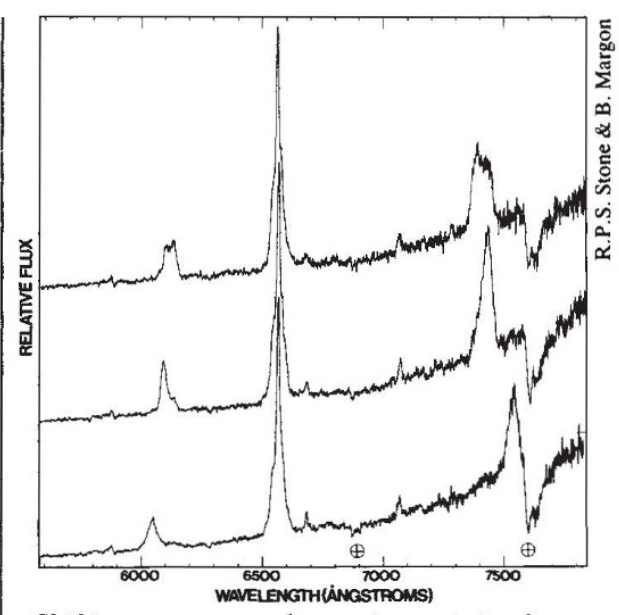

Shifting spectrum - the moving emission lines (here at about $6050 \AA$ and $7400 \AA$ ), the Dopplershifted reflections of the hydrogen-alpha feature at $6563 \AA$, which first indicated the bizarre properties of SS433.

spectrum of SS433, although not the changes in the emission lines. There were other astronomers and physicists who came close to making the key observations, but it was to Margon - who found, followed and identified the rapidly moving lines - that much of the glory went. Success came partly by luck, but largely from Margon's initiative and the ability to marshal the resources of several observatories equipped with large telescopes and blessed with good weather.

In The Quest for SS433, Clark tells the poignant and compelling tale of how close he came to revealing the secret of the star and how far others went in investigating it. It has been called a scientific detective story, but it also smacks of the thriller (although lacking a villain) as scientists make their way through international airports to observatories and meetings around the world, seeking clues to what Sky and Telescope later called the "enigma of the century".

Non-specialists can follow the story, but there are enough astrophysical data in the text and the illustrations to fascinate any scientist. Given the present job crisis in astronomy, this book should probably be kept out of the hands of bright young people - in case they should be unduly fired with enthusiasm for the subject.

Stephen $P$. Maran is a senior staff scientist in the Laboratory for Astronomy and Solar Physics, NASA - Goddard Space Flight Center, Greenbelt, Maryland 20771, USA. 